\title{
Effect of Osteopathic Cranial Manipulative Medicine on an Aged Rat Model of Alzheimer Disease
}

Hope Tobey, DO; Tyler Lucas, BS; Douglas Bledsoe, BS; Michael Mykins, BS; Caroline Campbell, BS; Stuart S. Berr, PhD; Todd Sasser, PhD; Richard Helm, PhD; Per Gunnar Brolinson, DO; Bradley G. Klein, PhD; Blaise M. Costa, MPharm, PhD

From Edward Via Virginia College of Osteopathic Medicine in Blacksburg (Drs Tobey, Brolinson, and Costa, Mr Bledsoe, Mr Mykins, and Ms Campbell); Virginia-Maryland College of Veterinary Medicine in

Blacksburg (Mr Lucas and Drs Klein and Costa); Virginia Tech in Blacksburg (Mr Lucas and Drs Klein and Costa); Department of Radiology and Medical Imaging at the University of Virginia School of Medicine in Charlottesville (Dr Berr); Bruker BioSpin Preclinical Imaging in Billerica, Massachusetts (Dr Sasser); and the Department of Biochemistry (Drs Helm and

Costa) at the School of Neuroscience at Virginia Tech (Dr Klein).

Financial Disclosures: Dr Sasser is an employee of the Bruker BioSpin, the manufacturer of the imaging system used in the study.

Support: This work was funded by Edward Via College of Osteopathic Medicine's Research Eureka Accelerator Program, Fund No. 10261 to Drs Costa, Tobey, Brolinson, and Klein. The imaging system was funded by $\mathrm{NIH}$ 1S10OD021672.

Address correspondence to Blaise M. Costa, MPharm, $\mathrm{PhD}$, Center for One Health Research, Virginia Tech, 1410 Prices Fork Rd, Blacksburg, VA 24060-3831.

Email: bcosta@vcom.vt.edu

Submitted

February 21, 2019; revision received April 2, 2019;

accepted April 22, 2019.
Context: In the aging brain, reduction in the pulsation of cerebral vasculature and fluid circulation causes impairment in the fluid exchange between different compartments and lays a foundation for the neuroinflammation that results in Alzheimer disease (AD). The knowledge that lymphatic vessels in the central nervous system play a role in the clearance of brain-derived metabolic waste products opens an unprecedented capability to increase the clearance of macromolecules such as amyloid $\beta$ proteins. However, currently there is no pharmacologic mechanism available to increase fluid circulation in the aging brain.

Objective: To demonstrate the influence of an osteopathic cranial manipulative medicine (OCMM) technique, specifically, compression of the fourth ventricle, on spatial memory and changes in substrates associated with mechanisms of metabolic waste clearance in the central nervous system using the naturally aged rat model of AD.

Results: Significant improvement was found in spatial memory in 6 rats after 7 days of OCMM sessions. Live animal positron emission tomographic imaging and immunoassays revealed that OCMM reduced amyloid $\beta$ levels, activated astrocytes, and improved neurotransmission in the aged rat brains.

Conclusion: These findings demonstrate the molecular mechanism of OCMM in aged rats. This study and further investigations will help physicians promote OCMM as an evidence-based adjunctive treatment for patients with AD.

J Am Osteopath Assoc. 2019;119(11):712-723. Published online October 15, 2019 doi:10.7556/jaoa.2019.121

Keywords: Alzheimer disease, amyloid- $\beta$, GFAP, neurotransmission, osteopathic cranial manipulative medicine, spatial memory, tau protein

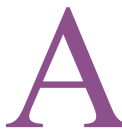
lzheimer disease (AD) is the sixth leading cause of death in the United States, with an estimated 5.3 million people across all ages having AD in $2015{ }^{1}$

Accumulation of metabolic wastes composed of extracellular deposits of amyloid- $\beta(A \beta)$ proteins and neurofibrillary tangles of tau protein are the hallmark pathologic findings in $\mathrm{AD}$. In the healthy brain, rapid exchange of cerebrospinal fluid (CSF) and interstitial fluid facilitates the clearance of macromolecules, including $A \beta$, from the brain parenchyma. $^{2}$ In the aging brain, reduction in the pulsation of cerebral vasculature and abnormal water channel expression cause significant impairment in fluid exchange., ${ }^{3,4}$ These modifications subsequently increase harmful protein concentrations in the interstitial fluid of the aging brain and pave a foundation for the neuroinflammation that results in $\mathrm{AD}$. The discovery of lymphatic vessels in the central nervous system (CNS) and their role in the clearance of brain-derived metabolic waste products ${ }^{5,6}$ opens an unprecedented capability to increase the clearance of macromolecules such as $\mathrm{A} \beta$ proteins. Limitations in 
improving CNS fluid circulation have restricted novel treatment strategies for age-related neurologic disorders in which excess metabolic waste accumulates in the brain. Currently there is no pharmacologic mechanism available to improve CNS vasculature pulsation or to increase fluid circulation in the aging brain. However, to our knowledge, increasing CNS fluid circulation and facilitation of the brain's lymphatic system by physical means has not yet been explored.

Improving lymphatic circulation is one of the therapeutic principles of osteopathic manipulative medicine (OMM). Animal studies demonstrate that lymphatic circulation in the peripheral nervous system can be improved by lymphatic pump treatment, an osteopathic manipulative technique. ${ }^{7-13}$ Furthermore, osteopathic cranial manipulative medicine (OCMM) has been routinely practiced to improve the symptoms of various neurologic disorders. ${ }^{10,14-21}$ Osteopathic cranial manipulative medicine may represent a means to physically facilitate CNS lymphatic and CSF circulation, potentially increasing the clearance of disease-inducing toxic metabolic waste. Examining the physiological and biochemical effects of these techniques may clarify this and other underlying mechanisms of improved clinical outcomes that may extend to the pathologic characteristics of AD.

A 3-dimensional modeling of the ventricular system of the rat brain confirms that its ventricular anatomy is similar to that of humans. ${ }^{22}$ Therefore, we hypothesized that fluid mobilization techniques to improve fourth ventricle circulation using the compression of the fourth ventricle (CV4) technique would be transferable to anesthetized rats. Because $95 \%$ of $\mathrm{AD}$ is sporadic and nonfamilial, ${ }^{23}$ we used an aged rat model. We sought to assess the mechanism of action for OCMM's potential improvement of AD-related clinical outcomes in 18-month-old, naturally aged rats.

\section{Methods}

\section{Animals}

All animal experimental procedures and animal housing were approved by the Institutional Animal
Care and Use Committee of Virginia Tech (protocol No. 15-099) and University of Virginia (protocol No. 3539). Twelve 18-month-old F344 male rats were obtained from Charles River Laboratories, Inc, and Envigo and randomly divided into 2 groups, (1) untreated (UT; n=6) and (2) OCMM (n=6). Six 16-week-old young adult rats were obtained from Charles River Laboratories, Inc. The young adult rats were not treated. All rats were provided with normal food and water ad libitum and housed with 12-hour light-dark cycle. All methods were performed in accordance with the relevant guidelines and regulations.

\section{OCMM Treatment}

One operator (H.T.) performed all OCMM sessions. The rats in the OCMM group received the CV4 technique every day for 7 days for 4 to 7 minutes at each session. Rats were anesthetized with $1.5 \%$ to $3 \%$ isoflurane throughout the procedure. Rats in the UT group were also anesthetized to nullify any influence of isoflurane in spatial learning. During the CV4 procedure, the operator applied mechanical pressure over the rat's occiput, medial to the junction of the occiput and temporal bone and inferior to the lambdoid suture to place tension on the dural membrane around the fourth ventricle. This gentle pressure was applied to resist cranial flexion with the aim of improving symmetry in the cranial rhythmic impulse (CRI), initiating a rhythmic fluctuation of the CSF, and improving mobility of the cranial bones and dural membranes. This rhythmic fluctuation is thought to be primarily due to flexion and extension that takes place at the synchondrosis between the sphenoid and basiocciput. The treatment end point was achieved when the operator identified that the tissues relaxed, a still point was reached, and improved symmetry or fullness of the CRI was felt. Currently, there is no quantitative measure for the pressure used in this treatment.

On day 1 of OCMM treatment, all 6 rats had significantly diminished or absent CRI, as well as increased firmness and tension of the soft tissue and bony structures palpated. Throughout the 7 days of treatment 
with the CV4 technique, increases in amplitude of the CRI with some persistent asymmetries were noted. However, by day 7, all 6 rats had significant softening of restricted tissues in the cranium and a fuller and symmetrical amplitude of the CRI. One rat was noted to still have a slightly restricted CRI at the end of day 7; however, it was noted to be much less restricted then before the initiation of treatment on day 1 . These palpatory changes would be clinically interpreted as an indication of improved somatic dysfunction and, therefore, improved function of bone and soft tissues and presumed improvement in arterial, venous, and lymphatic circulation.

\section{Spatial Learning and Memory Assay}

The Morris water maze (MWM) assay was performed to study the spatial learning and memory in both groups. On day 0 , rats were exposed to the MWM for acclimatization. ${ }^{24}$ The maze is a circular washtub with a 5-ft diameter and 2-ft high walls. The water maze was divided into 4 quadrants electronically by a flexible video tracking system (AnyMaze 5.1, Stoelting Co.) with a circular platform (5-in diameter) in the northwest quadrant. The tank was filled with room temperature water to about $1 \mathrm{~cm}$ below the platform for training trials. Four visual cues with large designs were drawn on paper and attached to the edge of the maze. These landmarks were visible to the rats during all trials.

During training trials (days 1-4), rats were trained from 4 quadrants (northwest, northeast, southeast, and southwest) every day for 4 days. During training, the water was transparent and the platform visible to the animal. During each trial, a rat was trained to reach the platform from 1 of the 4 different quadrant starting points. A maximum of 60 seconds was allotted to reach the platform; in case a rat failed to reach the platform within 60 seconds, it was manually guided to the platform. Before each training trial, the rat was placed on the platform for 15 seconds to let it observe the cues around the water maze and their spatial relationship to the platform. After 15 seconds, the animal was gently placed in the water at one of the quadrants with its head facing the wall. At this point, video recording began, and the quadrant was noted. After reaching the platform, the rat was allowed to be on the platform for 15 seconds before starting the next trial. After completing all 4 trials for the day, rats were gently wiped dry and placed on the warming pad for 2 minutes before being returned to the home cage.

During probe trials (days 5-8), water was opaque and the platform was invisible to the animal. Nontoxic tempera paint was used to make the water opaque, and the water was elevated to submerge the platform about $1 \mathrm{~cm}$ below the water surface. Two probe trials were performed for each animal every day from different quadrants. In the probe trial, the animal had to remember the platform's spatial location with reference to the surrounding environment to reach the platform as quickly as possible. After day- 8 trials, rats were transported tothe University of Virginia Molecular Imaging Core for positron emission tomography (PET)

\section{Positron Emission Tomography}

A US Food and Drug Administration-approved compound, florbetapir F18, which binds to $A \beta$ plaques, was used as a radiotracer. Four hundred to $500 \mu \mathrm{Ci}$ of tracer dissolved in 300 to $500 \mu \mathrm{L}$ of $0.9 \%$ isotonic sodium chloride solution was administered through a single bolus injection into the tail vein 45 to 60 minute before imaging. A high-resolution scanner used for PET, single-photon emission computed tomography, and computed tomography (CT) was used for imaging. Imaging was performed while rats were anesthetized and placed in the prone position at the center of the scanner bed. After PET scanning, a CT scan of the whole cranium was performed. The CT images served as templates to analyze the PET signals and were used for attenuation correction of the PET images. Based on the PET images, volume of interest was identified with the guidance of the Schiffer rat brain atlas 25 provided in the PMOD software (V 3.8, PMOD Technologies LLC) as described by Balsara et al. ${ }^{26}$ Thirty-three different brain regions, including the forebrain, hippocampus, and entorhinal cortex were included, and 
florbetapir F18 standard uptake values were calculated for each region. After PET scanning on day 9, rats were euthanized by cervical dislocation before harvesting brain tissue for biochemical analysis. Rats that were not imaged were euthanized at the Virginia Tech Phase IV facility.

\section{Immunohistochemical Analysis}

One-half of the hemisphere of each brain was used for immunohistochemical (IHC) analysis, and the other half was saved for other immunoassays. For IHC, brain tissues were immersed in $4 \%$ paraformaldehyde in phosphate-buffered saline (PBS) overnight and transferred to $30 \%$ sucrose-PBS solution until they floated. Approximately 16- $\mu \mathrm{m}$ sagittal sections were obtained using cryostat and stored at $-80^{\circ} \mathrm{C}$ until used. For experiments, tissue sections were equilibrated to room temperature for 15 minutes and washed 3 times, 5 minutes each time with PBS, and blocked at room temperature for 1 hour using a blocking buffer $(5 \%$ goat serum, 3\% bovine serum albumin (BSA), 0.1\% Triton-X 100 in PBS). Chicken anti-GFAP (glial fibrillary acidic protein) (1:500; Abcam), rabbit anti-vesicular glutamate transporter-1 (VGluT1; 1:250; Synaptic Systems) and immunoglobulin G2a (IgG2a) mouse anti-glutamate decarboxylate-67 (GAD67 1:500; Millipore) were diluted in blocking buffer and applied to tissue sections before allowing them to react overnight at $4^{\circ} \mathrm{C}$. Sections were then washed 3 times, 5 minutes each time with $\mathrm{PBS}$, before probing with appropriate fluorophore conjugated secondary antibodies, goat anti-chicken, $647 \mathrm{~nm}$ (Abcam); goat anti-IgG2a mouse, $568 \mathrm{~nm}$ (ThermoFisher); goat-anti-rabbit 488nm (Abcam) with 1:1000 dilution in blocking buffer for 1 hour at room temperature. Slides were washed with PBS 3 times for 5 minutes each time and mounted in vectashield DAPI mounting medium.

\section{Data Collection and Analysis}

Immunostained tissue sections were imaged using a $\times 40$ water immersion objective on a Zeiss LSM 880 confocal microscope. Super resolution images were obtained using the Zeiss Airyscan detector. Airyscan projections were analyzed using ImageJ integrated density analysis. Density analysis was calculated by establishing a threshold for the intensity of each channel and by measuring the average intensity value and multiplying it by the area occupied in that threshold. An individual threshold was set for each channel in order to subtract out the background.

\section{Western Blot}

One-millimeter-thick coronal sections of brain tissue from UT and OCMM rats were collected from the region dorsal to the prefrontal cortex using a brain matrix. Tissue samples were lysed using a lysis and extraction buffer with a protease inhibitor cocktail (ThermoFisher No. 89900 and 78440), and protein concentration was determined using a Bradford assay. Equal amounts (30 $\mu \mathrm{g}$ unless stated otherwise) of protein samples from each group were electrophoresed in sodium dodecyl sulfate polyacrylamide gels (10\%) under reducing conditions followed by transfer to polyvinylidene difluoride (PVDF) (pore size, $0.2 \mu \mathrm{m}$ ) membranes. Blots were blocked with 5\% nonfat dry milk in Tris-buffered saline with $0.075 \%$ Tween 20 and probed with antibodies recognizing A $\beta 42$. Synthetic rat A $\beta 42$ peptide (Anaspec, No. AS-25381) has been used as a standard marker for antibody recognition and molecular weight for the bands observed in the Western blots assay. Two monoclonal antibodies recognizing N-terminal (Abcam, No. ab201060) or C-terminal (Cell Signaling, No. 14974) end of A $\beta 42$ peptide were used, in addition to a polyclonal (Abcam, No. ab10148) antibody to probe the synthetic and native $\mathrm{A} \beta$ peptides in the Western blot assay. Antibodies recognizing glial fibrillary acidic protein (GFAP; chicken), aquaporin-4 (AQP4), lymphatic vessel endothelial hyaluronan receptor 1 (LYVE1; rabbit, ThermoFisher), GAD67 (mouse IgG2a, Millipore) and vGluT1 (rabbit, SYnaptic SYstems) were used as described in the Results section. The secondary antibodies used were goat anti-rabbit/chicken/ mouse IgG (GeneTex). Chemiluminescence (Pierce) 
signals were detected by a Flurochem M (Protein Simple) scanner.

\section{Statistical Analysis}

Statistical significance was determined at $P<.05, P<.01$, and $P<.001$ using a 2 -tailed unpaired $t$ test. Values given represent mean (SE).

\section{Results}

\section{OCMM Improves Spatial Learning and Memory in Naturally Aged Rats}

The MWM results showed that the UT 16-week-old young adult rats reached the platform significantly quicker (shorter escape latency) compared with the UT aged rats in all 8 days of trial. This finding indicates age-dependent reduction in spatial memory (Figure 1B-D). The rats treated with OCMM exhibited a significantly shorter escape latency than the UT rats on days 4,5 , and 6 of the 8-day experiment. During probe trials, the platform was not visible. This factor excludes the possibility that the OCMM-treated rats could have reached the platform quicker because of improved vision. The underlying assumption of the MWM assay is that rodents do not like to be wet; therefore, when exposed to water, they would try to find an escape platform as quickly as possible. On the first of the 4 day- 1 trials, no animal could reach the platform within 60 seconds, so they were then manually guided to the platform. However, on day 1 during trials 2 to 4 , some rats reached the platform in less than 60 seconds.

On the day- 4 training trial and probe trial day 5 and day 6, OCMM-treated rats had a statistically significant shorter escape latency than the UT group. Further, we have studied more parameters, including distance travelled, number of entries into the platform zone (NW quadrant), and time spent in the platform zone on day 4. Since day 4 was the last day of training trials and the first day to exhibit a statistically significant difference between the UT and OCMM groups, we presented these parameters on day 4. Untreated young adult rats were significantly different (Figure 1D) from the UT aged rats in all parameters studied on day 4 . Total distance travelled and the distance travelled inside the NW quadrant were not significantly different between the UT and OCMM-treated rats Figure 1D). However, UT rats made a significantly higher number of entries into the platform quadrant and spent more time in that quadrant compared with OCMM-treated rats. The video shows the UT and OCMM rat movements. Fewer entries and less time in the target zone reveal that OCMM-treated rats could more successfully identify the platform location when they were proximal to the platform, despite traveling in and around the platform zone as much as UT rats.

\section{Live Imaging Reveals a Numerical Reduction of A $\beta$ Proteins in OCMM-Treated Rats}

To validate the outcomes from the behavioral studies, PET was performed on day 9. The PET techniques have recently made it possible to evaluate brain $A \beta$ deposition in patients with AD. The results revealed that compared with UT rats, OCMM-treated rats had an overall reduction in the florbetapir F18 signal in whole brain tissue (Figure 2). The order of 3 regions where maximum reduction in florbetapir F18 signals were observed was midbrain, ventral tegmental area, and hippocampus. These findings from live animal imaging suggest an increased clearance of $\mathrm{A} \beta$ proteins from the brains of OCMM-treated rats. It was anticipated that the mechanical pressure (Figure 1 A) applied on the surface of the rat head increased glymphatic substrates that could have improved the fluid dynamics in the aged rat brain. This pressure may have ultimately improved the clearance of $A \beta$ protein from the brain through one of the recently identified meningeal lymphatic vessels that are perhaps involved in CNS waste clearance. ${ }^{5,6,27}$

\section{OCMM Alters A $\beta$ levels in Association With Astrocyte Activation and Upregulation of} Aquaporin and Lymphatic Vessels

To understand the OCMM-induced improvement in learning and memory, total $A \beta$ protein levels were 

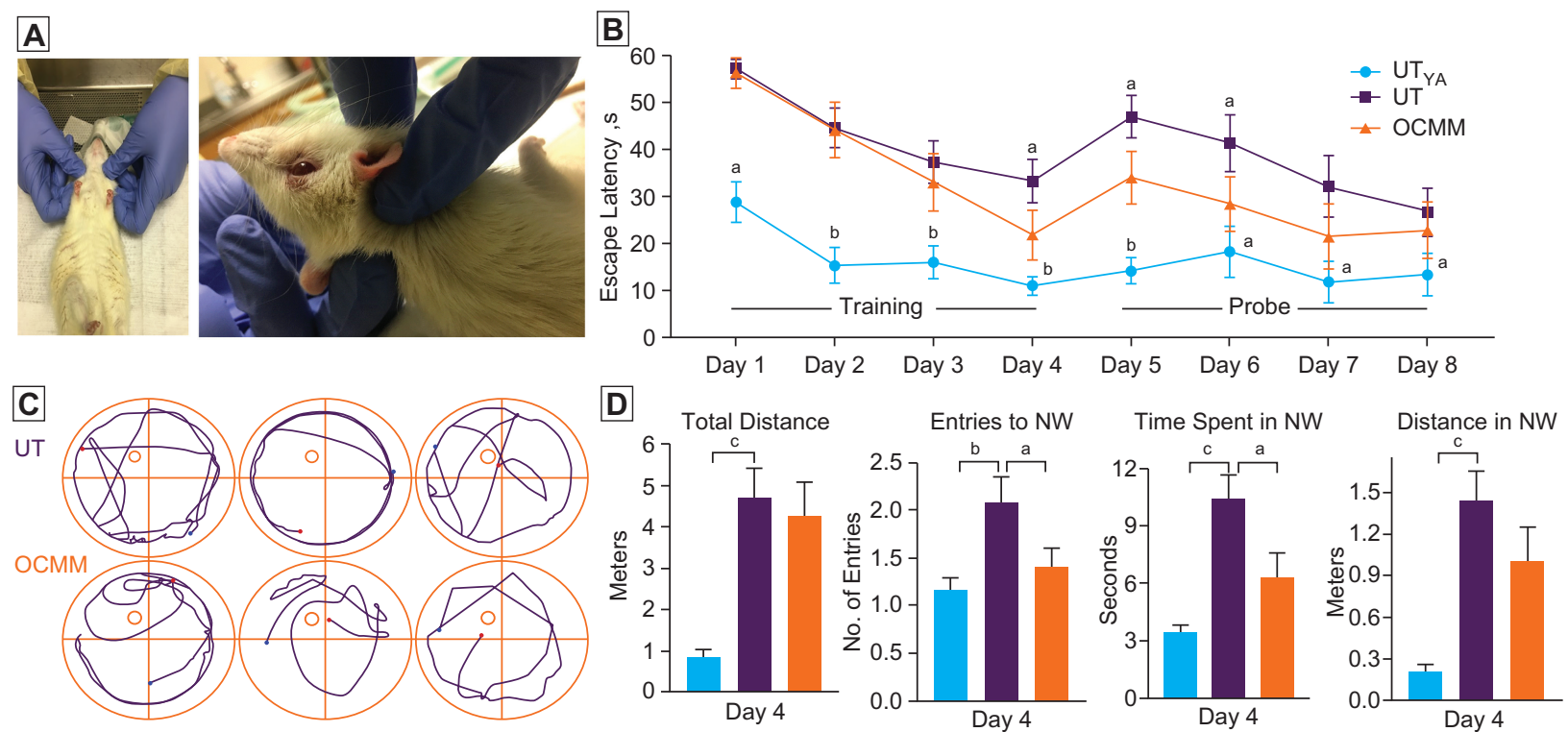

Figure 1.

(A) Position of the anesthetized animal when performing osteopathic cranial manipulative medicine (OCMM) and a closer view of OCMM performance. (B) Time taken to reach platform on each experiment day. Rats received 4 days (days 1-4, platform visible) of training before probe trials (days $5-8$, platform invisible). Sixteen-week-old untreated young adult (UT $\mathrm{Ya}_{\mathrm{Ya}}$ ) male rats exhibited significantly shorter escape latency on day 1-8 compared with untreated (UT) aged rats. During training day 4 and probe days 5 and 6, OCMM-treated rats reached the platform sooner than the UT rats. (C) Representative plots show the trajectory of UT and OCMM-treated animal movement. Four quadrants, platform location (circle at northwest quadrant), starting (blue dot), and end (red dot) points are marked. (D) Histograms show the distance travelled, number of entries to the northwest quadrant (NW), time spent in NW, and distance travelled in NW on the last day (day 4) of the training trial period. $\mathrm{n}=6$; unpaired, 2-tailed, $t$ test, ${ }^{\mathrm{a}} P<.05,{ }^{\mathrm{b}} P<.01$, and ${ }^{\mathrm{c}} P<.001$.

studied in the rat brain lysate. Western blots were performed with denatured samples to detect all $A \beta$ protein that can exist in the native state as monomeric $A \beta$ fragments, intermediate-length $\mathrm{A} \beta$ with intact $\mathrm{C}$-terminal domain, an uncleaved native amyloid precursor protein, and an oligomer of $A \beta$ fragments. After titrating with 1,10 , and $100 \mathrm{ng}$ of synthetic $\mathrm{A} \beta 42$ peptides, $100 \mathrm{ng}$ concentration was chosen to use as standard and probed with all 3 different antibodies. Both N-terminal and C-terminal monoclonal antibodies recognized the synthetic rat A $\beta 42$ peptide around $4 \mathrm{kDa}$ (Figure 3A and B). The other antibody (polyclonal, No. ab10148) did not detect the synthetic $A \beta 42$ peptide. Therefore, the Western blot results obtained from this antibody are not included in Figure 3.

The manufacturer claims that the monoclonal N-terminal antibody (ab201060) recognizes a conformation-dependent, aggregation-selective epitope of $A \beta$ at amino acid position 3-6 (sequence: EFRH). This antibody preferentially binds to sodium dodecyl sulfate-resistant oligomers over monomer on Western blots, ${ }^{28}$ and these signals were detected at $\sim 37 \mathrm{kDa}$ and $\sim 4 \mathrm{kDa}$. Consistent with the claims made by the manufacturer, this antibody detected a $\sim 37-\mathrm{kDa}$ band in all samples with varying intensity (Figure 3A). Furthermore, this antibody also detected a $\sim 4-\mathrm{kDa}$ signal in aged rat brain lysate samples. Thus, both $\sim 37-\mathrm{kDa}$ and $\sim 4-\mathrm{kDa}$ signals represent variable-length fragments of $A \beta$ protein or $A \beta 42$ oligomer, which is resistant to chemical and heat denaturation steps. Nonetheless, synthetic A $\beta 42$ peptide band was detected at the anticipated $\sim 4 \mathrm{kDa}$ (Figure 3A), and there was no higher-molecular-weight band found at this sample running lane. The absence of a GAPDH band at this lane confirms that there was no contamination of brain lysate sample. The average A $\beta 42$ signal from the brain 


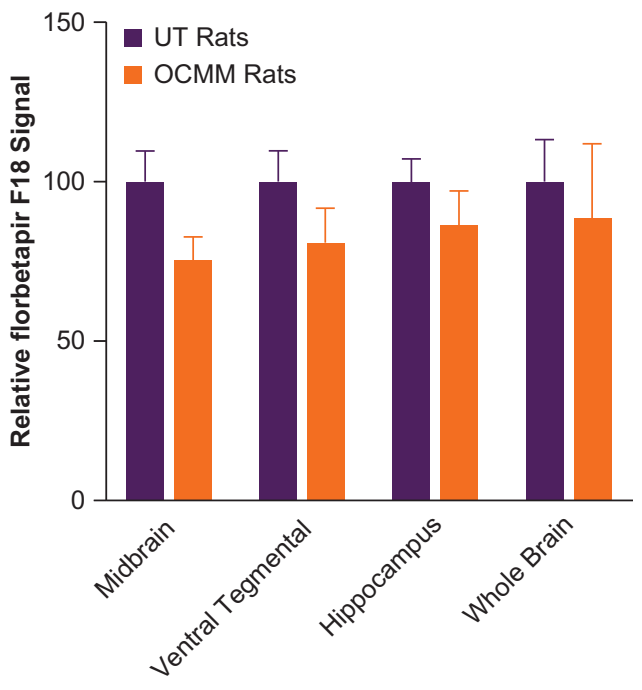

Figure 2.

Histograms show the relative florbetapir F18 signal in untreated (UT; n=3) and osteopathic cranial manipulative medicine (OCMM)-treated $(n=3)$ aged rats. Normalized and averaged (mean [SE]) signals revealed a numerical reduction in amyloid $\beta(A \beta)$ levels at midbrain $(24.5 \%$ [2.4\%]), ventral tegmental area (19.1\% [2.6\%]), hippocampus $(13.4 \%$ [1.6\%]), and whole brain $(11.2 \%$ [2.9\%]) of OCMM-treated UT rats.

tissue lysate of the UT young adult rats (Figure 3A) was significantly less than the UT rats in both $\mathrm{N}$-terminal and $\mathrm{C}$-terminal antibodies.

The $\mathrm{C}$-terminal domain antibody detected the signal for synthetic A $\beta 42$ peptide at the correct molecular weight, $\sim 4 \mathrm{kDa}$. Furthermore, this antibody detected a stronger $\sim 4-\mathrm{kDa}$ signal (Figure 3B) at the lysateloaded lanes. Perhaps a weak but measureable $\sim 37-\mathrm{kDa}$ band was also detected by this antibody. Consistent signals of $\mathrm{A} \beta$ at around $4 \mathrm{kDa}$ and $37 \mathrm{kDa}$ obtained from different antibodies binding at opposing ends of the $A \beta$ peptide reveal that these signals are variable-length $A \beta$ fragments but not false-positives. These observations reinforce the notion that $A \beta$ protein may exist as variable-length fragments in the rat brain. Altogether, the results obtained from all 3 antibodies are in agreement with each other that OCMM-treated rats had less $\mathrm{A} \beta$ protein compared with the age-matched UT rats.
The A $\beta$ Western blot data suggest that OCMM may have facilitated the clearance of potentially toxic forms of $A \beta$ proteins.

It was hypothesized that there should have been an increase in waste clearance mechanisms, in which astrocytes and water channels could be directly involved, as reviewed recently. ${ }^{27}$ To support this, quantitative immunoassays were performed to determine the level of GFAP, a marker for astrocytes and AQP4, which is a water channel predominantly expressed in the astrocyte end feet processes ${ }^{29}$ reduction of which exacerbates brain $A \beta$ accumulation and memory deficits. ${ }^{30,31}$ Results from the Western blots done with the brain tissue lysates reveal that OCMM-treated rats had a significant increase in GFAP and AQP4 expression compared with the UT group (Figure 3C). Furthermore, the IHC assay results demonstrated an increase in GFAP expression in the dentate gyrus (Figure 3J). Increased astrocyte representation combined with the increased expression of AQP4 channels motivated us to study the marker for lymphatic vessels (LYVE1) and that also exhibited a significant increase in OCMM-treated rats (Figure 3J). These observations indicate that there could have been an increase in glymphatic system substrates and that may account for the apparent increased clearance of $A \beta$ proteins, which is known to improve learning and memory. ${ }^{32,33}$

\section{OCMM Modulates Synaptic Transmission}

\section{Through Excitatory and Inhibitory Pathways}

To study other potential underlying mechanisms of improvement in learning and memory after OCMM, immunoassays were performed to determine the level of presynaptic glutamatergic and $\gamma$-aminobutyric acidergic neuronal markers, vGlut1 and GAD67, respectively. Immunohistochemical assays results of the sagittal section of brain tissue supported the Western blot finding that the relative puncta density for vGlut1 was significantly elevated in OCMM-treated rats compared with UT rats in the hippocampal CA1 region (Figure 4). Additionally, Western blot results showed that the levels of vGlut1 and GAD67 were significantly 


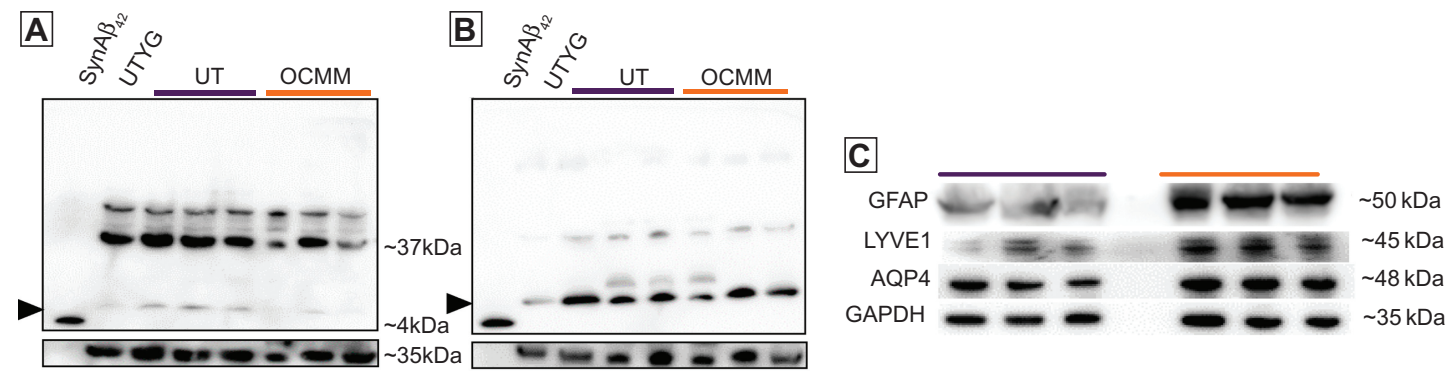

D

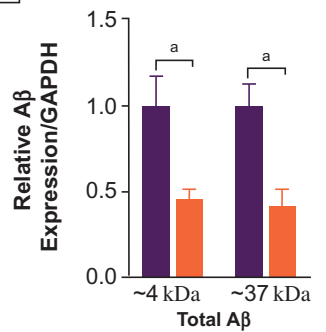

E

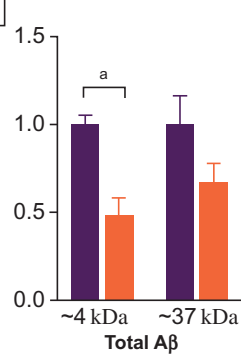

$\mathrm{F}$

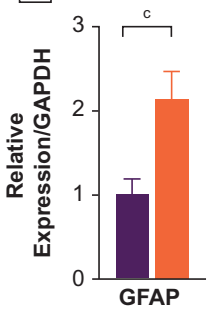

G

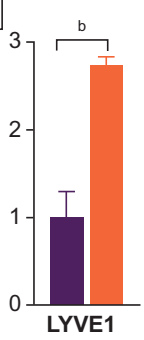

H

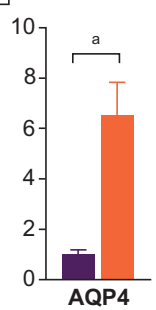

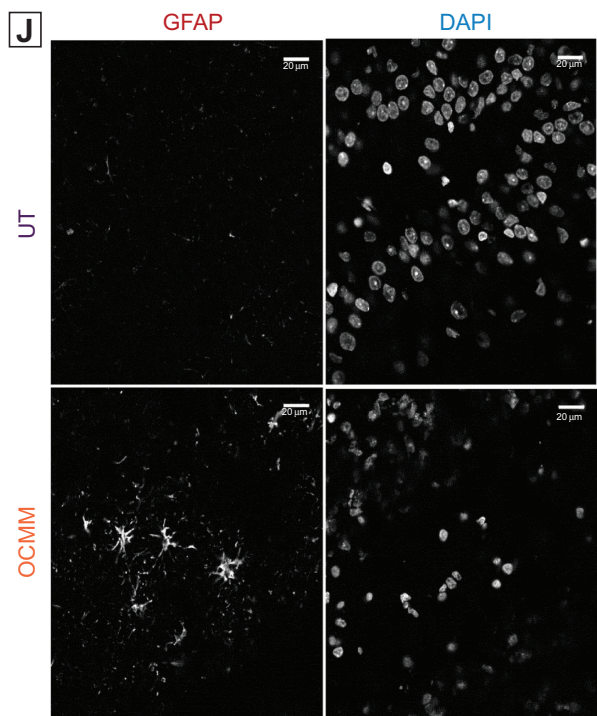

GFAP+DAPI
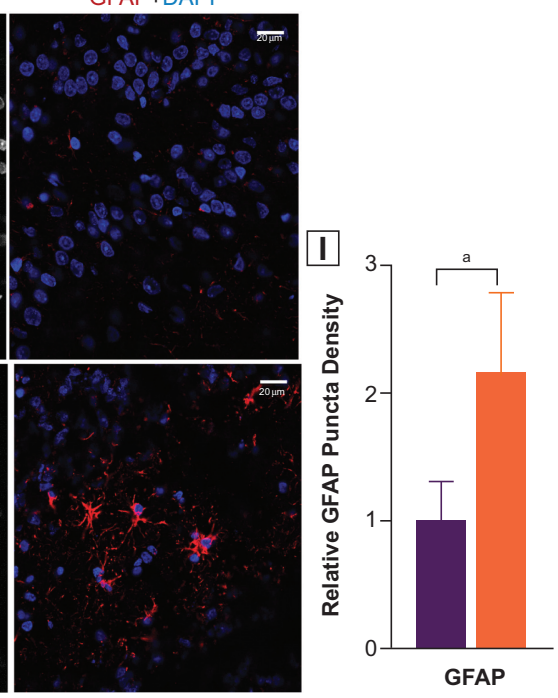

Figure 3.

(A, B) Representative whole-gel pictures of Western blot assays show the total amyloid $\beta(A \beta)$ motif containing proteins as probed with 2 monoclonal anti-A $\beta 42$ primary antibodies recognizing N-terminal (Abcam, No. ab201060 [A]) and C-terminal (Cell Signaling, No. $14974[B])$ end of the $A \beta 42$ peptide. SynA $\beta_{42}$, control synthetic rat $A \beta_{42}$ peptide (Anaspec No. AS-25381). Untreated young adult (UT $\mathrm{Ya}_{\mathrm{a}}$ ), control rat brain lysate. Arrowheads indicate the expected $\sim 4-\mathrm{kDa}$ size $A \beta_{42}$ peptide. Higher-molecular-weight $(\sim 37 \mathrm{kDa})$ bands indicate the presence of variable length of amyloid $C$-terminal domain fragments with intact $A \beta_{42}$ motif. Relative density analysis revealed that rats treated with osteopathic cranial manipulative medicine (OCMM; $\mathrm{n}=3)$ had significantly less $(P<.05,100 \mu \mathrm{g}$ of total protein was loaded) anticipated $\sim 4-\mathrm{kDa}$ $\mathrm{A} \beta_{42}$ peptide and $\sim 37 \mathrm{kDa}$ fragments in $\mathrm{N}$-terminal ([D] $P<.05$ for $\sim 4 \mathrm{kDa}, P<.05$ for $\sim 37 \mathrm{kDa}$ ) and C-terminal ([E] $P<.05$ for $\sim 4 \mathrm{kDa}$ ) recognizing anti-A $\beta_{42}$ antibodies. (C) Western blots showing total glial fibrillary acidic protein (GFAP), aquaporin-4 (AQP4), and lymphatic vessel endothelial hyaluronic receptor 1 (LYVE1) expression in aged untreated (UT) and OCMM-treated rats; GAPDH served as a control. All Western blots were repeated with duplicate samples of each animal brain lysate. $(\mathrm{F}-\mathrm{H})$ Histograms of relative density analysis reveal that OCMM-treated rats had significantly higher GFAP $(P<.001)$, AQP4 $(P<.05)$, and LYVE1 $(P<.01)$ expression than the UT rats $(P<.05)$. (J) Immunohistochemical assay shows the increased expression of GFAP at the dentate gyrus in OCMM-treated rats. (I) Histograms represent the normalized averages and statistical significance. Unpaired 2-tailed $t$ test, ${ }^{\mathrm{a}} P<.05,{ }^{\mathrm{b}} P<.01,{ }^{\mathrm{c}} P<.001$. Scale bar, $20 \mu \mathrm{M}$. 

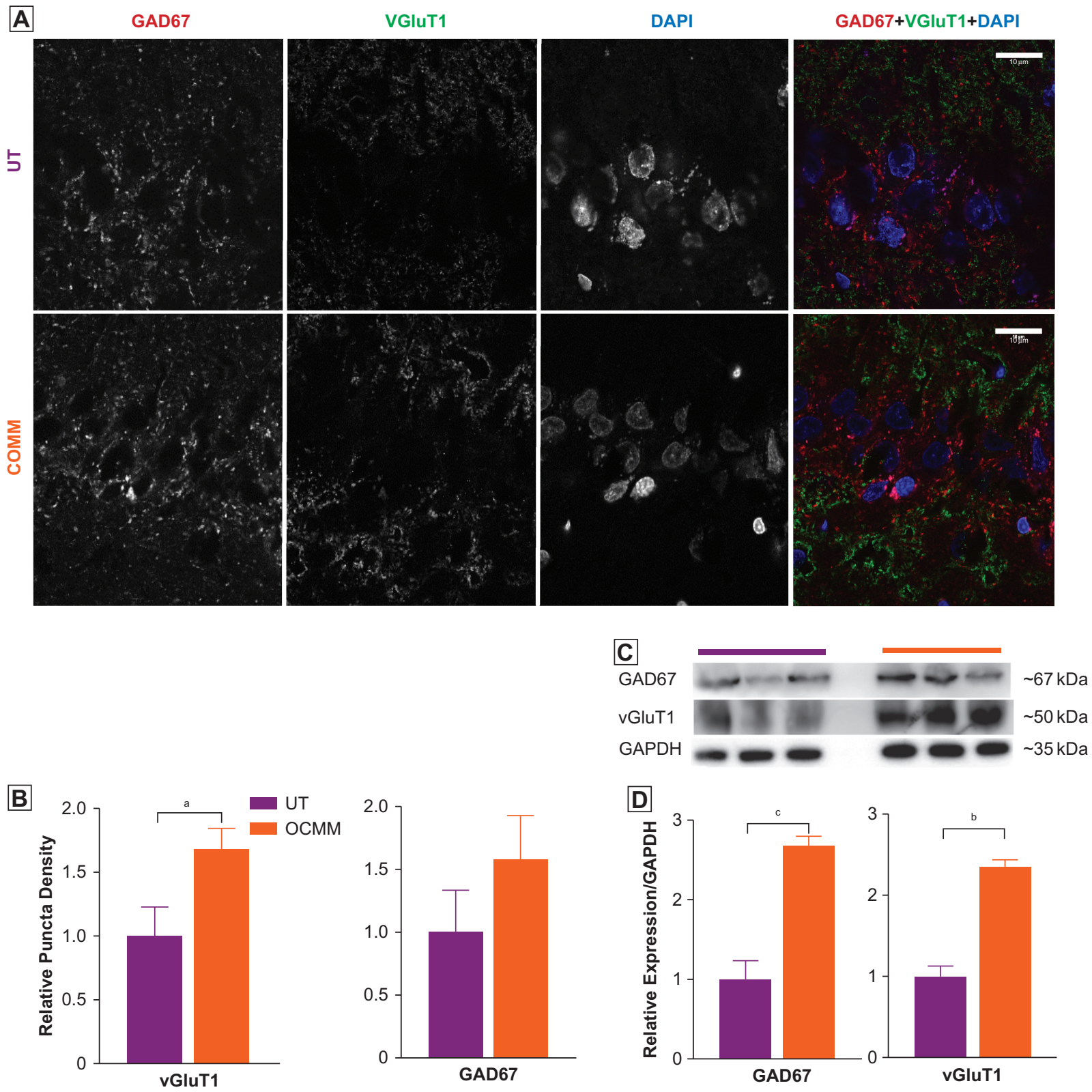

Figure 4.

(A) Representative immunohistochemical images of the hippocampal CA1 region in untreated (UT; n=3) and osteopathic cranial manipulative medicine (OCMM)-treated $(n=3)$ rat brain sagittal sections immunostained for glutamic acid decarboxylase (GAD67), vesicular glutamate transporter 1 (VGLUT1), and 4,6-diamidino-2-phenylindole (DAPI). (B) Puncta density analysis reveals significantly increased expression of VGLUT1 but not GAD67 in the CA1 region (mean [SEM] puncta density in UT, 1.0 [0.33] vs OCMM, 1.57 [0.34]; $P<.05$ ). (C) Western blots show the increased expression of GAD67. GAD67 (UT relative density, 1.00 [0.23] vs OCMM, 2.68 [0.11]; $P<.001$ ) and (D) VGLUT1 (UT relative density, 1.00 [0.12] vs OCMM, 2.35 [0.07]; $P<.01$ ). Histograms represent the normalized averages. Unpaired 2 -tailed $t$ test, ${ }^{a} P<.05$, ${ }^{\mathrm{b}} P<.01,{ }^{\mathrm{c}} P<.001$. Scale bar, $10 \mu \mathrm{M}$. 
increased in OCMM-treated rats compared with UT rats (Figure 4C and D). An increase in vGluT1 expression that is involved in the vesicular storage of glutamate suggests that OCMM may have also altered glutamatergic synaptic transmission either independently or as a cause of improved $A \beta$ clearance. An insignificant elevation of GAD67 expression in the hippocampal CA1 region may indicate a stronger excitatory component of excitatory-inhibitory balance mechanisms associated with hippocampal long-term potentiation. ${ }^{34,35}$ However, GAD67 expression was significantly increased in the prefrontal cortex, a region involved in spatial memory, which corroborates the significant increase in GAD67 expression observed in the Western blot (Figure 4C).

\section{Discussion}

The majority of osteopathic physicians practice as primary care physicians and provide medical care for older patients. ${ }^{36}$ Osteopathic physicians who practice OMM use these techniques to improve the symptoms of various disorders. ${ }^{10,14-21}$ Although clinically effective, the underlying mechanism for the effect of OCMM is not well understood. The knowledge on glymphatic and meningeal lymphatic systems in the brain lends credence to the theory that OCMM improves CNS fluid circulation. However, there remains a void in scientific evidence supporting the capability of OCMM techniques to alter fluid dynamics and lymphatic circulation in the brain or other underlying mechanisms of improved clinical outcomes. If OCMM is capable of improving CNS fluid circulation, it could represent a minimally invasive means to take advantage of the CNS glymphatic system to overcome the age-related deficits in cerebrovascular fluid circulation and metabolic exchange that may be contributing to AD.

Manipulation-induced skull deflections have been extensively studied in live nonhuman primates, ${ }^{37,38}$ dogs ${ }^{39}$ cats,${ }^{40}$ rabbits, ${ }^{41}$ and unembalmed human cadaver heads. ${ }^{42-44}$ These studies have revealed that a mechanical pressure can cause cranial deflections up to $910 \mu \mathrm{m}$ based on the region of the skull. ${ }^{45}$ These cranial deflections may influence fluid circulation through the glymphatic periarterial influx and meningeal lymphatic perivenous efflux pathways that run close to the cranial bones. The function of glymphatic and meningeal lymphatic pathways is largely regulated by the water channels (AQP4) expressed in the perivascular astrocyte end-foot processes. ${ }^{27}$ This is in agreement with a significant increase in AQP4 protein expression in the OCMM-treated rats compared with UT rats (Figure 3H). This finding, combined with the other biochemical and behavioral changes we reported, supports the notion that OCMM-induced cranial bone deflections can potentially improve fluid circulation.

\section{Limitations}

In this pilot study, we could not directly measure the OCMM-induced changes in CNS fluid dynamics. This limitation was due to lack of funding associated with expensive animals, reagents, and equipment involved to study the fluid dynamics. We actively seek support from state and federal agencies to study the changes in CNS fluid dynamics and associated molecular mechanisms.

\section{Future Directions}

In the present study using an OCMM technique, we demonstrated improved spatial learning and memory in the aged rat model of AD. This improvement is connected to changes in indicators of increased brain metabolic waste clearance and changes in the markers of altered neurotransmission. At this point, it is unclear how these 2 types of changes are related. An immediate extension of these findings would be to study the effect of OCMM on the pharmacokinetics of medications that are currently used for the treatment of patients with $\mathrm{AD}$ and other neurologic disorders.

\section{Conclusion}

The current study found that OCMM improved spatial memory and reduced cerebrovascular waste products 
in the brains of rats. Cranial bone deflections induced by OCMM may improve fluid circulation, which in turn could improve the metabolic waste clearance in the aged rat model of AD. Further investigations are required to validate the current experimental findings in human participants.

\section{Acknowledgment}

We acknowledge Tullia Johnston, BS, and Bryanna Vacca, BS, for critical reading of this manuscript.

\section{Author Contributions}

All authors provided substantial contributions to conception and design, acquisition of data, or analysis and interpretation of data; Drs Tobey, Brolinson, Klein, and Costa drafted the article or revised it critically for important intellectual content; Dr Costa gave final approval of the version of the article to be published; and all authors agree to be accountable for all aspects of the work in ensuring that questions related to the accuracy or integrity of any part of the work are appropriately investigated and resolved.

\section{References}

1. Alzheimer's disease facts and figures. J Alzheimer Assoc. 2017;13 (4):325-373. doi:10.1016/j.jalz.2017.02.001

2. Iliff, JJ, Wang M, Liao Y, et al. A paravascular pathway facilitates CSF flow through the brain parenchyma and the clearance of interstitial solutes, including amyloid beta. Sci Transl Med. 2012;4 (147):147ra111. doi:10.1126/scitransImed.3003748

3. Iliff JJ, Wang M, Zeppenfeld DM, et al. Cerebral arterial pulsation drives paravascular CSF-interstitial fluid exchange in the murine brain. J Neurosci. 2013;33(46):18190-18199.

4. Zieman SJ, Melenovsky V, Kass DA. Mechanisms, pathophysiology, and therapy of arterial stiffness. Arterioscler Thromb Vasc Biol. 2005;25(5):932-943.

5. Aspelund A, Antila S, Proulx ST, et al. A dural lymphatic vascular system that drains brain interstitial fluid and macromolecules. J Exp Med. 2015;212(7):991-999. doi:10.1084/jem.20142290

6. Louveau A, Smirnov I, Keyes TJ, et al. Structural and functional features of central nervous system lymphatic vessels. Nature. 2015;523(7560):337-341. doi:10.1038/nature14432

7. Creasy C, Schander A, Orlowski A, Hodge LM. Thoracic and abdominal lymphatic pump techniques inhibit the growth of $S$ pneumoniae bacteria in the lungs of rats. Lymphat Res Biol. 2013;11 (3):183-186. doi:10.1089//rb.2013.0007

8. Hodge LM. Osteopathic lymphatic pump techniques to enhance immunity and treat pneumonia. Int J Osteopath Med. 2012;15 (1):13-21.

9. Hodge LM, Bearden MK, Schander A, et al. Lymphatic pump treatment mobilizes leukocytes from the gut associated lymphoid tissue into lymph. Lymphat Res Biol. 2010;8(2):103-110. doi:10.1089/ Irb.2009.0011
10. Hodge LM, Creasy C, Carter K, Orlowski A, Schander A, King HH. Lymphatic pump treatment as an adjunct to antibiotics for pneumonia in a rat model. J Am Osteopath Assoc. 2015;115(5):306-316. doi:10.7556/jaoa.2015.061

11. Huff JB, Schander A, Downey HF, Hodge LM . Lymphatic pump treatment augments lymphatic flux of lymphocytes in rats. Lymphat Res Biol. 2010;8(4):183-187. doi:10.1089//rb.2010.0009

12. Schander A, Downey HF, Hodge LM. Lymphatic pump manipulation mobilizes inflammatory mediators into lymphatic circulation. Exp Bio Med (Maywood). 2012;237(1):58-63. doi:10.1258/ebm.2011.011220

13. Schander A, Padro D, King HH, Downey HF, Hodge LM. Lymphatic pump treatment repeatedly enhances the lymphatic and immune systems. Lymphat Res Biol. 2013;11(4):219-226. doi:10.1089/ Irb.2012.0021

14. Bordoni B, Zanier E. Sutherland's legacy in the new millennium: the osteopathic cranial model and modern osteopathy. Adv Mind Body Med. 2015;29(2):15-21.

15. Cerritelli, F, Martelli M, Renzetti C, Pizzolorusso G, Cozzolino V, Barlafante G. Introducing an osteopathic approach into neonatology ward: the NE-O model. Chiropr Man Therap. 2014;22:18. doi:10.1186/ 2045-709X-22-18

16. Fraix M, Gordon A, Graham V, Hurwitz E, Seffinger MA. Use of the SMART Balance Master to quantify the effects of osteopathic manipulative treatment in patients with dizziness. J Am Osteopath Assoc. 2013;113(5):394-403.

17. Genese JS. Osteopathic manipulative treatment for facial numbness and pain after whiplash injury. J Am Osteopath Assoc. 2013;113 (7):564-567. doi:10.7556/jaoa.2013.008

18. Miana, L, Bastos VH, Machado S, et al. Changes in alpha band activity associated with application of the compression of fourth ventricular (CV-4) osteopathic procedure: a qEEG pilot study. J Bodyw Mov Ther. 2013;17(3):291-296. doi:10.1016/j.jbmt.2012.10.002

19. Mulcahy J, Vaughan B. Sensations experienced and patients perceptions of osteopathy in the cranial field treatment. J Evid Based Complementary Altern Med. 2014;19(4):235-246. doi:10.1177/ 2156587214534263

20. Pizzolorusso G, Cerritelli F, D'Orazio M, et al. Osteopathic evaluation of somatic dysfunction and craniosacral strain pattern among preterm and term newborns. J Am Osteopath Assoc. 2013;113(6):462-467.

21. Rolle G, Tremolizzo L, Somalvico F, Ferrarese C, Bressan LC. Pilot trial of osteopathic manipulative therapy for patients with frequent episodic tension-type headache. J Am Osteopath Assoc. 2014;114 (9):678-685. doi:10.7556/jaoa.2014.136

22. Nie B, Chen K, Zhao S, et al. A rat brain MRI template with digital stereotaxic atlas of fine anatomical delineations in paxinos space and its automated application in voxel-wise analysis. Hum Brain Mapp. 2013;34(6):1306-1318. doi:10.1002/hbm.21511

23. Braidy N, Poljak A, Jayasena T, Mansour H, Inestrosa NC, Sachdev PS. Accelerating Alzheimer's research through 'natural' animal models. Curr Opin Psychiatry. 2015;28(2):155-164. doi:10.1097/ YCO.0000000000000137

24. Vorhees CV, Williams MT. Morris water maze: procedures for assessing spatial and related forms of learning and memory. Nat Protoc. 2006;1(2):848-858.

25. Schiffer WK, Mirrione MM, Biegon A, Alexoff DL, Patel V, Dewey SL. Serial microPET measures of the metabolic reaction to a microdialysis probe implant. J Neurosci Methods. 2006;155(2):272-284. 
26. Balsara RD, Chapman SE, Sander IM, et al. Non-invasive imaging and analysis of cerebral ischemia in living rats using positron emission tomography with 18F-FDG. J Vis Exp. 2014(94). doi:10.3791/51495

27. Louveau A, Plog BA, Antila S, Alitalo K, Nedergaard M, Kipnis J. Understanding the functions and relationships of the glymphatic system and meningeal lymphatics. J Clin Invest. 2017;127 (9):3210-3219. doi:10.1172/JCI90603

28. Hatami, A, Albay R III, Monjazeb S, Milton S, Glabe C. Monoclona antibodies against Abeta42 fibrils distinguish multiple aggregation state polymorphisms in vitro and in Alzheimer disease brain. $J$ Biol Chem. 2014;289(46):32131-32143. doi:10.1074/jbc.M114.594846

29. Sofroniew MV Vinters HV., Astrocytes: biology and pathology. Acta Neuropathol. 2010;119(1):7-35. doi:10.1007/s00401-009-0619-8

30. Szu JI, Binder DK. The role of astrocytic aquaporin-4 in synaptic plasticity and learning and memory. Front Integr Neurosci. 2016;10:8 doi:10.3389/fnint.2016.00008

31. Xu Z, Xiao N, Chen Y, et al. Deletion of aquaporin-4 in APP/PS1 mice exacerbates brain Abeta accumulation and memory deficits. Mol Neurodegener. 2015;10:58.

32. Wang J, Ho L, Chen L, et al. Valsartan lowers brain beta-amyloid protein levels and improves spatial learning in a mouse model of Alzheimer disease. J Clin Invest. 2007;117(11):3393-3402.

33. Lord A, Englund $H$, Söderberg L, et al. Amyloid-beta protofibril levels correlate with spatial learning in Arctic Alzheimer's disease transgenic mice. FEBS J. 2009;276(4):995-1006. doi:10.1111/ j.1742-4658.2008.06836.x

34. Froemke RC. Plasticity of cortical excitatory-inhibitory balance. Annu Rev Neurosci. 2015;38:195-219. doi:10.1146/ annurev-neuro-071714-034002

35. Bonansco C, Fuenzalida M. Plasticity of hippocampal excitatory-inhibitory balance: missing the synaptic control in the epileptic brain. Neural Plast. 2016;2016:8607038. doi:10.1155/2016/ 8607038

36. Licciardone JC. A national study of primary care provided by osteopathic physicians. J Am Osteopath Assoc . 2015;115 (12):704-713. doi:10.7556/jaoa.2015.145

37. Michael DK, Retzlaff EW. A preliminary study of cranial bone movement in the squirrel monkey. J Am Osteopath Assoc. 1975;74 (9):866-869

38. Retzlaff EW, Michael DK, Roppel RM. Cranial bone mobility. J Am Osteopath Assoc. 1975;74(9):869-873.

39. Oudhof HA, van Doorenmaalen WJ. Skull morphogenesis and growth hemodynamic influence. Acta Anat (Basel). 1983;117(3):181-186.

40. Adams T, Heisey RS, Smith MC, Briner BJ. Parietal bone mobility in the anesthetized cat. J Am Osteopath Assoc. 1992;92(5):599-600, 603-610, 615-622.

41. Downey PA, Barbano T, Kapur-Wadhwa R, Sciote JJ, Siegel MI, Mooney MP. Craniosacral therapy: the effects of cranial manipulation on intracranial pressure and cranial bone movement. J Orthop Sports Phys Ther. 2006;36(11):845-853.

42. Yoganandan N, Pintar FA, Sances A Jr, et al. Biomechanics of skull fracture. J Neurotrauma. 1995;12(4):659-668.

43. Stalnaker RL, Fogle JL. Driving point impedance characteristics of the head. J Biomech. 1971;4(2):127-139. doi:10.1016/0021-9290(71) 90023-6

44. Franke E.K. Response of the human skull to mechanical vibrations J Acoustic Soc Am. 1956;28(6):1277-1284.

45. Seimetz, CN. Kemper AR, Duma SM. An investigation of cranial motion through a review of biomechanically based skull deformation literature. Int J Osteopath Med. 2012;15(4):152-165

() 2019 American Osteopathic Association 